\title{
The Hippo pathway transcriptional co-activator, YAP, confers resistance to cisplatin in human oral squamous cell carcinoma
}

\author{
KYOHEI YOSHIKAWA $^{1 *}$, KAZUMA NOGUCHI $^{1 *}$, YOSHIRO NAKANO $^{2}$, MICHIYO YAMAMURA $^{1}$, \\ KAZUKI TAKAOKA ${ }^{1}$, TOMOKO HASHIMOTO-TAMAOKI ${ }^{2}$ and HIROMITSU KISHIMOTO ${ }^{1}$ \\ Departments of ${ }^{1}$ Oral and Maxillofacial Surgery and ${ }^{2}$ Genetics, Hyogo College of Medicine, \\ Nishinomiya, Hyogo 663-8501, Japan
}

Received January 28, 2015; Accepted March 2, 2015

DOI: 10.3892/ijo.2015.2948

\begin{abstract}
Cisplatin (CDDP) is widely used to treat oral squamous cell carcinoma (OSCC), however, many patients exhibit acquired drug resistance. Yes-associated protein (YAP) is a transcriptional co-activator of the Hippo pathway that regulates organ size and promotes cell proliferation. YAP overexpression correlates with epithelial-mesenchymal transition and nodal metastasis, resulting in anti-tubulin drug resistance. Whether YAP overexpression is the cause of CDDP resistance in cancer cells is unclear, therefore, we investigated the correlation between YAP expression and CDDP sensitivity. We established three CDDP-resistant cell lines (OSC-19-R, SCCKN-R and HSC-3-R) from the OSCC parental cell lines. We also examined the expression levels of ATP7B, GST- $\pi$ and ERCC1, which are strongly associated with CDDP resistance, and Hippo pathway-related proteins by western blotting. Using immunocytochemistry, we examined the cellular localization of YAP. Additionally, following knockdown of YAP using short interfering RNAs (siRNAs), we analyzed changes in sensitivity to CDDP. Compared with parental OSC-19 cells, OSC-19-R cells were obviously larger. Expression levels of YAP were not significantly different between OSC-19 and OSC-19-R. However, expression levels of phosphorylated YAP in OSC-19-R were decreased. We observed translocation of YAP from the cytoplasm to the nucleus in OSC-19-R cells. Knockdown of YAP using siRNAs revealed that sensitivity to CDDP was significantly increased. Translocation of YAP
\end{abstract}

Correspondence to: Dr Kazuma Noguchi, Department of Oral and Maxillofacial Surgery, Hyogo College of Medicine, 1-1 Mukogawa-cho, Nishinomiya, Hyogo 663-8501, Japan

E-mail: knoguchi@hyo-med.ac.jp

*Contributed equally

Abbreviations: OSCC, oral squamous cell carcinoma; CDDP, cisplatin; SCCHN, squamous cell carcinoma of the head and neck; EMT, epithelial-mesenchymal transition; DMEM, Dulbecco's modified Eagle's medium

Key words: oral squamous cell carcinoma, cisplatin, acquired resistance, Hippo pathway, Yes-associated protein correlated with the acquisition of CDDP resistance. YAP could be a new therapeutic target for the treatment of patients with cancer that are resistant to CDDP.

\section{Introduction}

Despite recent advances in therapeutic treatments using multimodal therapies, including the excision of malignant tissue combined with radio- and chemotherapy, high oral squamous cell carcinoma (OSCC) mortality rates of $\sim 50 \%$ have not improved over time (1). The main reason for this is that early lymph node metastasis of cancer cells is difficult to detect $(2,3)$. When induction chemotherapy with cis-diamminedichloroplatinum (II) (CDDP) and 5-fluorouracil was introduced for treatment of squamous cell carcinoma of the head and neck (SCCHN) in the early 1980s, response rates of $30-40 \%$ were initially reported $(4,5)$. A problem that emerged during OSCC treatment was increased resistance against many of the approved chemotherapeutic agents, such as paclitaxel, docetaxel, cetuximab and CDDP (6).

CDDP is used to treat a range of malignancies, including SCCHN. CDDP resistance studied in cultured cancer cells has shown that cellular defense mechanisms in a highly complex pleiotropic phenotype confers resistance by reducing apoptosis, upregulating DNA damage repair mechanisms, altering cellcycle checkpoints, and disrupting assembly of the cytoskeleton (7). Alterations to the cytoskeleton disrupt cellular protein trafficking, and redirect transporters away from the cell surface. This results in cells that are permanently resistant to CDDP, and also resistant to other compounds that usually enter into cells via uptake transporters. The pleiotropic mechanisms underlying CDDP resistance are well described but poorly understood in their entirety and in terms of clinical significance $(7,8)$.

The Hippo pathway was initially identified for sensing and regulating organ size in Drosophila and mammals (9). In the Hippo pathway, YAP is a transcriptional co-activator that participates in several context-dependent transcriptional programs that regulate organ size and promote cell proliferation (10). YAP was proposed as a candidate oncogene and its dysregulation associated with hepatocellular carcinoma, non-small cell lung carcinoma, esophageal squamous cell carcinoma, ovarian cancer and gastric cancer $(9,11-14)$. YAP is also reported to cause epithelial-mesenchymal transition 
(EMT), stimulate proliferation, inhibit apoptosis, and to promote tumor progression in a tissue-specific manner $(9,12)$. Ge et al reported that YAP expression in primary SCCHN was associated with nodal metastasis due to EMT (15). Zhao et al identified YAP as an important player in response to apoptosis of cancer cells induced by the anti-tubulin drug paclitaxel (16). Whether YAP overexpression confers CDDP resistance to cancer cells is unclear, therefore we sought to determine whether this was the case.

\section{Materials and methods}

Cell culture and establishment of CDDP-resistant cell lines. OSCC cell lines were maintained on $100-\mathrm{mm}$ plates at $37^{\circ} \mathrm{C} / 5 \%$ $\mathrm{CO}_{2}$, and grown as monolayers in Dulbecco's modified Eagle's medium (DMEM), supplemented with L-glutamine, penicillin, streptomycin and 10\% (v/v) fetal bovine serum (FBS). The OSC-19 cell culture was provided by the Kanazawa University Graduate School of Medical Science (Kanazawa, Japan). CDDP was purchased from Nichi-Iko Pharmaceutical Co. Ltd. and stored as a $0.5 \mathrm{mg} / \mathrm{ml}$ stock solution in $0.9 \%(\mathrm{w} / \mathrm{v})$ $\mathrm{NaCl}$ at room temperature and shielded from light.

CDDP-resistant variants of the OSCC cell line were isolated using stepwise selection by increasing the concentration of CDDP. To generate the resistant cells the starting CDDP concentration was $0.5 \mu \mathrm{g} / \mathrm{ml}$; once cultures became confluent in medium containing CDDP, its concentration was increased to $1.0,1.5,2.0,2.5$ or $3.0 \mu \mathrm{g} / \mathrm{ml}$. Cells were continuously exposed to CDDP for 4-8 weeks at each concentration, with culture medium refreshed every 3 days. Cultures were passage once a week over a 40-week period. Established CDDP-resistant cell cultures were designated OSC-19-R, SCCKN-R, and HSC-3-R, and maintained in the presence of CDDP.

Growth assays and determination of CDDP sensitivity. The sensitivity of cells to CDDP was determined by also using the Cell Counting Kit-8 (Dojindo, Kumamoto, Japan). On day 1, cells $\left(5.0 \times 10^{3}\right.$ cells/well in $100 \mu 1$ of DMEM) were seeded in 96-well plates and cultured at $37^{\circ} \mathrm{C}$. On day 2 , the medium was replaced with $100 \mu \mathrm{l}$ of fresh DMEM containing 0.33-167 $\mu \mathrm{M}$ CDDP and cells were allowed to incubate for 3 days. On day 5 , $10 \mu \mathrm{l}$ of Cell Counting Kit- 8 solution was added; after $3 \mathrm{~h}$, the absorbance in each well was measured at $450 \mathrm{~nm}$ using a microplate spectrophotometer (Bio-Rad, Hercules, CA, USA). The fold increase or decrease in resistance was determined by dividing the $\mathrm{IC}_{50}$ value of CDDP for resistant cells with that for parental cells.

Western blotting. Cell lysates were subjected to western blotting as described previously (17). The primary antibodies used were rabbit polyclonal antibodies against YAP, phospho-YAP (serine-127), TEAD, LATS1, LATS2, AKT, and phospho-AKT (serine-473) (Cell Signaling Technology, Boston, MA, USA), glutathione S-transferase (GST)- $\pi$ (Calbiochem, USA), ATP7B, ERCC1, P73 (Santa Cruz Biotechnology, Santa Cruz, CA, USA). We used a goat polyclonal antibody against actin (Santa Cruz Biotechnology) as an internal control. The secondary antibodies we used were anti-goat and anti-rabbit immunoglobulin Gs (IgGs) conjugated with alkaline phosphatase (Santa Cruz Biotechnology).
Table I. Sensitivity of CDDP for each of the cell lines we used and generated. ${ }^{\mathrm{a}}$

\begin{tabular}{lccc}
\hline Cell line & $\begin{array}{c}\mathrm{IC}_{50} \text { in } \mu \mathrm{M} \\
(\text { mean } \pm \mathrm{SD})\end{array}$ & $\begin{array}{c}\text { Fold resistance to } \\
\text { CDDP compared with } \\
\text { parental cell line }\end{array}$ & p-value \\
\hline OSC-19 & $17.1 \pm 12.7$ & - & $<0.01$ \\
OSC-19-R & $113.3 \pm 17.6$ & 6.6 & \\
SCCKN & $1.7 \pm 0.3$ & - & $<0.01$ \\
SCCKN-R & $9.8 \pm 0.8$ & 5.7 & $<0.01$ \\
HSC-3 & $9.9 \pm 0.9$ & - & \\
HSC-3-R & $54.6 \pm 4.5$ & 5.5 & \\
\hline
\end{tabular}

${ }^{a}$ The fold resistance was determined by comparing $\mathrm{IC}_{50}$ values for CDDP in resistant cells to that in parental cells.

Immunocytochemistry. The primary antibodies we used in our immunocytochemistry analysis included rabbit anti-YAP (Cell Signaling Technology) and a mouse polyclonal antibody against human actin (Santa Cruz). Cultured cells were fixed in $3.7 \%$ paraformaldehyde for $20 \mathrm{~min}$ at room temperature. After blocking with $2 \%(\mathrm{w} / \mathrm{v})$ bovine serum albumin (BSA), cells were treated with a primary antibody at $4^{\circ} \mathrm{C}$ overnight. Cells were washed, and then incubated with an anti-rabbit IgG conjugated to fluorescein isothiocyanate (FITC) or an anti-mouse IgG conjugated to rhodamine phalloidin (Cytoskeleton, Denver, CO, USA). Cells were then stained with 4,6-diamidino-2-phenylindole (DAPI). Fluorescence images were obtained using a confocal laser-scanning microscope (LSM 510 version 3.2; Carl Zeiss Co. Ltd., Oberkochen, Germany).

Transfection of siRNAs. Transfection of siRNAs was conducted as described previously (17). Cells were cultured in DMEM supplemented with $10 \%$ FBS for $24 \mathrm{~h}$ and transfected with $5 \mu \mathrm{M}$ siRNA using Thermo Scientific DharmaFECT Transfection reagents (Roche, Indianapolis, IN, USA) according to the manufacturer's instructions. SMART pool siRNAs targeting YAP (L-012200-00-0020) and control siRNA, and On-Target plus GAPDH (D-001830-01-20) were purchased from Dharmacon Inc. (Lafayette, CO, USA).

Statistical analysis. Data are expressed as means \pm SD. Results were analyzed, and individual group means were compared using Student's t-test. A p-value of $<0.05$ was considered statistically significant.

\section{Results}

CDDP-resistant cell lines. When the CDDP concentration was raised after a step, $\sim 30 \%$ cell death occurred. After $\sim 1$ month, OSCC cells acquired resistance to CDDP step-by-step. The CDDP-resistant cell lines we generated from SCCKN, OSC-19, and HSC-3 were designated SCCKN-R, OSC-19-R, and HSC-3-R (Fig. 1A). The CDDP $\mathrm{IC}_{50}$ for each cell line was determined using an MTT assay (Table I); the SCCKN-R, OSC-19-R, and HSC-3-R cell lines were 5.7-, 6.6- and 5.5-fold more resistant to CDDP than their parental cells, respectively. 
A

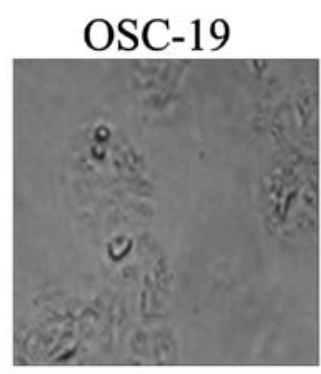

SCCKN

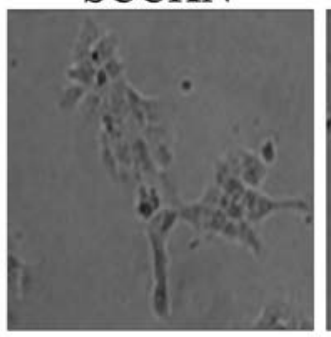

OSC-19-R

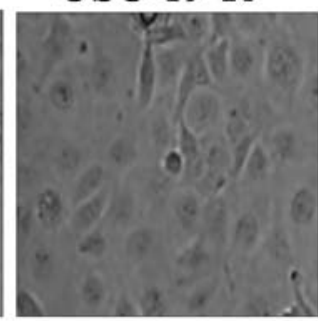

SCCKN-R

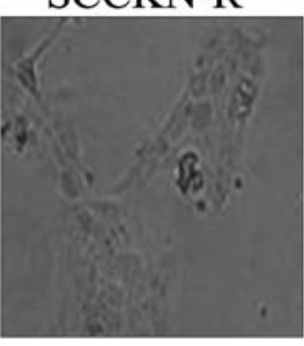

HSC-3

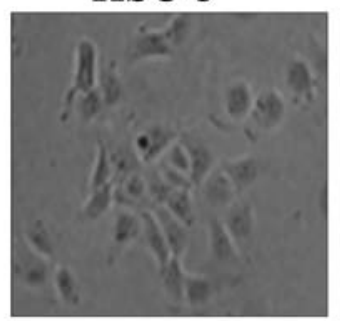

B
HSC-3-R
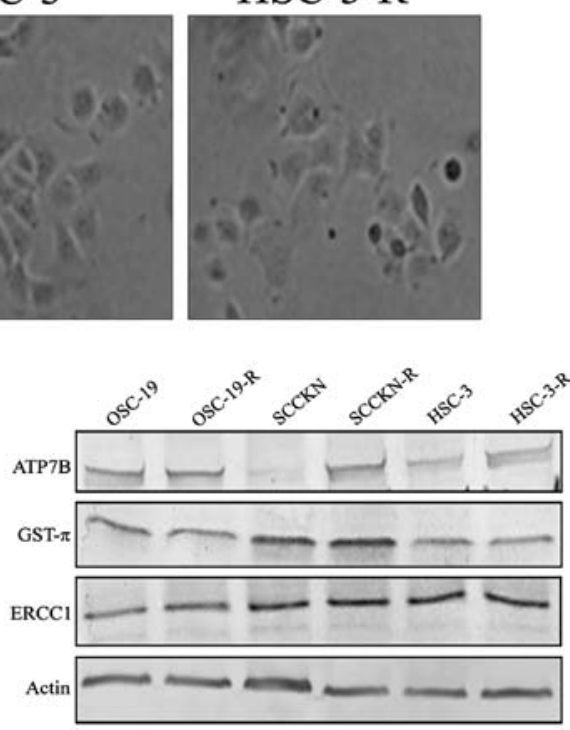

Figure 1. (A) The CDDP-resistant cell lines generated from SCCKN, OSC-19 and HSC-3 were designated SCCKN-R, OSC-19-R and HSC-3-R. (B) Western blot analysis for each cell line, showing expression of ATP7B, GST- $\pi$ and ERCC1. The SCCKN-R and HSC-3-R cells lines overexpressed ATP7B. There were no obvious difference between parental and CDDP-resistant cell lines with respect to expression of GST- $\pi$ and ERCC1.

A

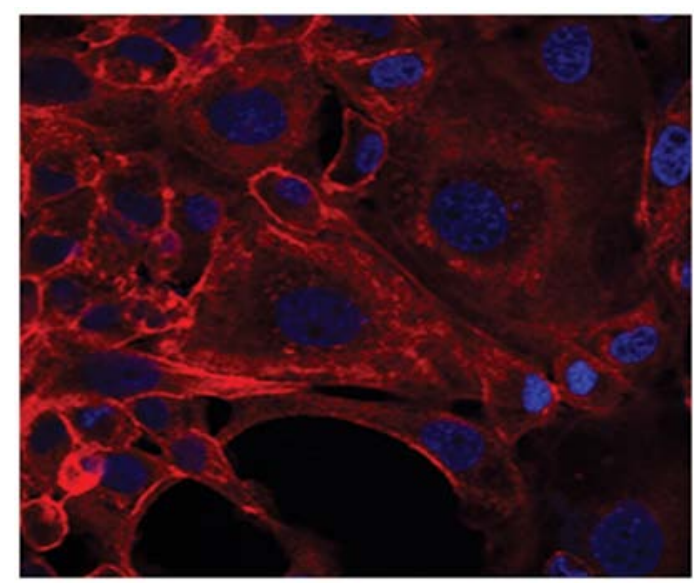

\section{C}

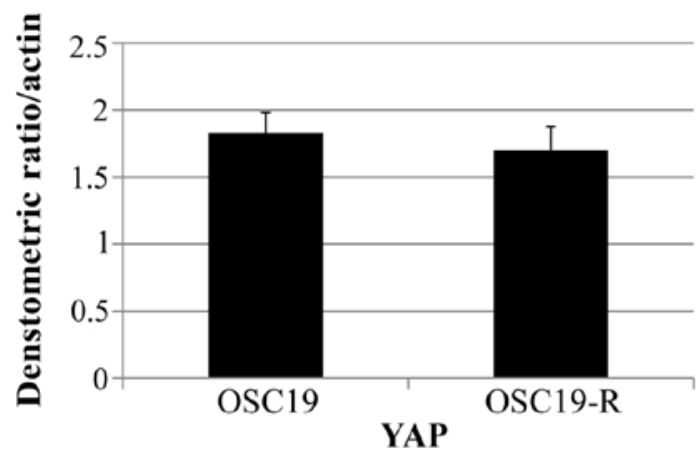

B
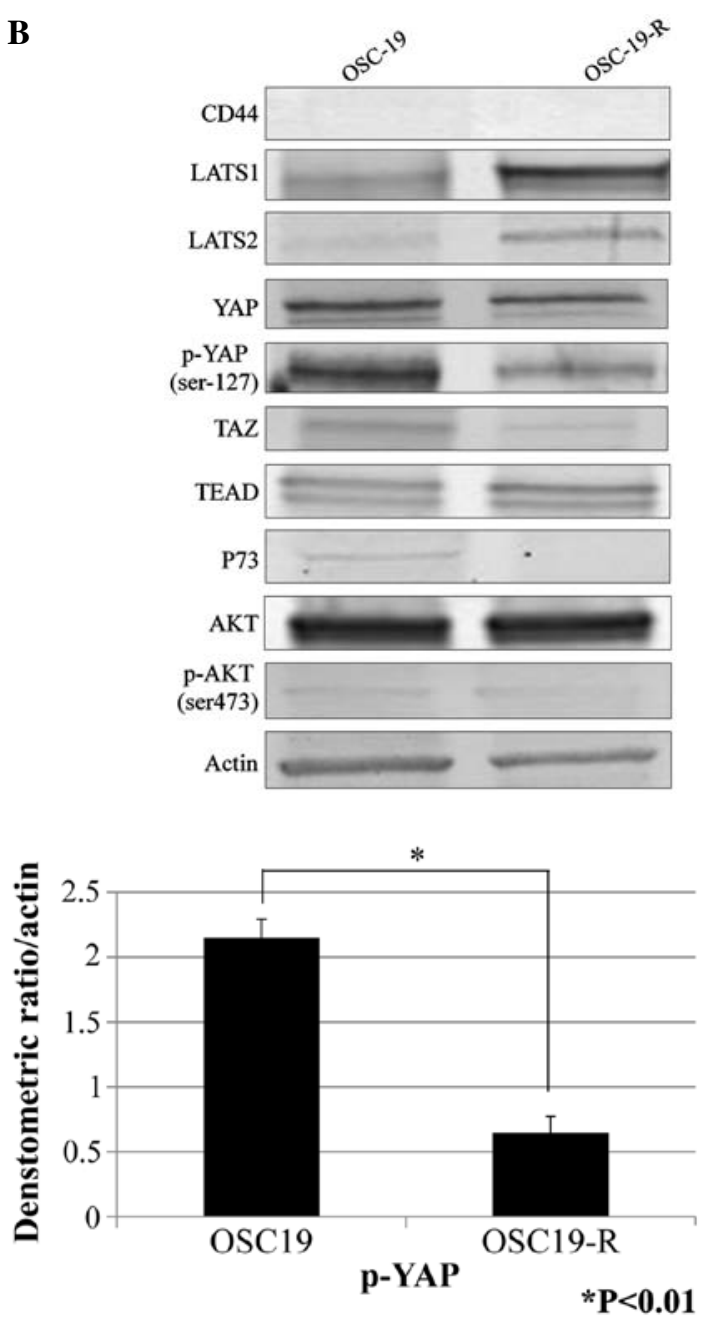

Figure 2. (A) Immunocytochemical analysis of mixed populations of OSC-19 and OSC-19-R cells. Blue, nucleus (DAPI); red, actin (phalloidin). OSC-19-R cells were obviously larger than OSC-19 cells. (B and C) Western blot analysis of Hippo pathway proteins. Expression levels of YAP were not significantly different between OSC-19 and OSC-19-R cells. Expression of phosphorylated YAP in OSC-19-R cells was clearly decreased compared with that in OSC-19 cells. Expression levels of LATS1/2 in OSC-19-R cells was increased compared with that seen in OSC-19 cells. 

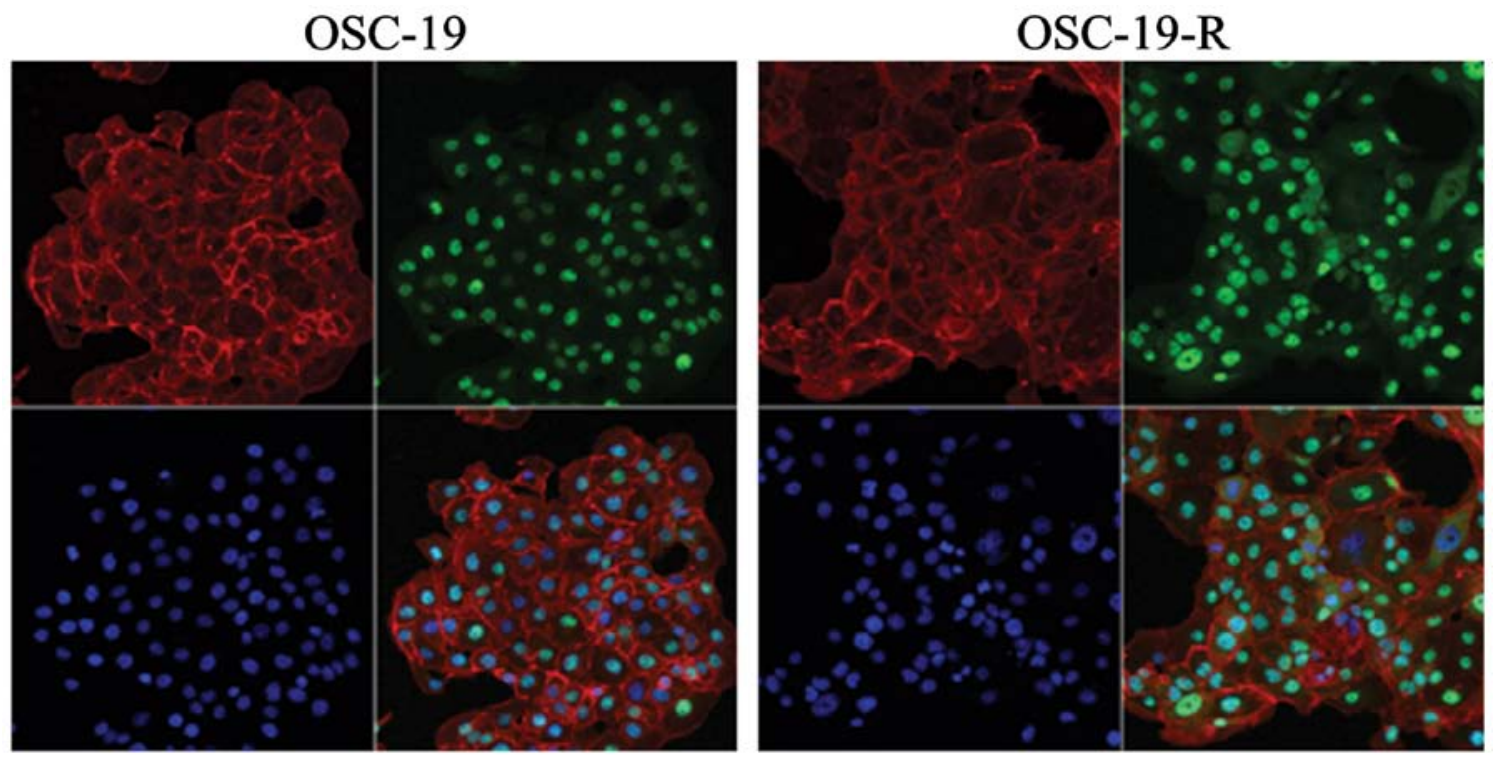

Figure 3. Immunocytochemical analysis of YAP cellular localization. Blue, nucleus (DAPI); red, actin (phalloidin); and green, YAP (FITC). Higher YAP expression in the nucleus was observed in OSC-19-R cells compared with that in parental cells.

A

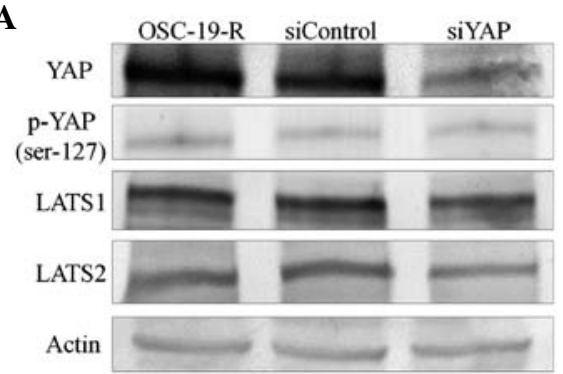

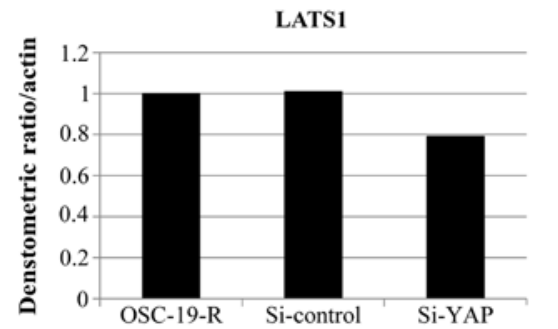

B
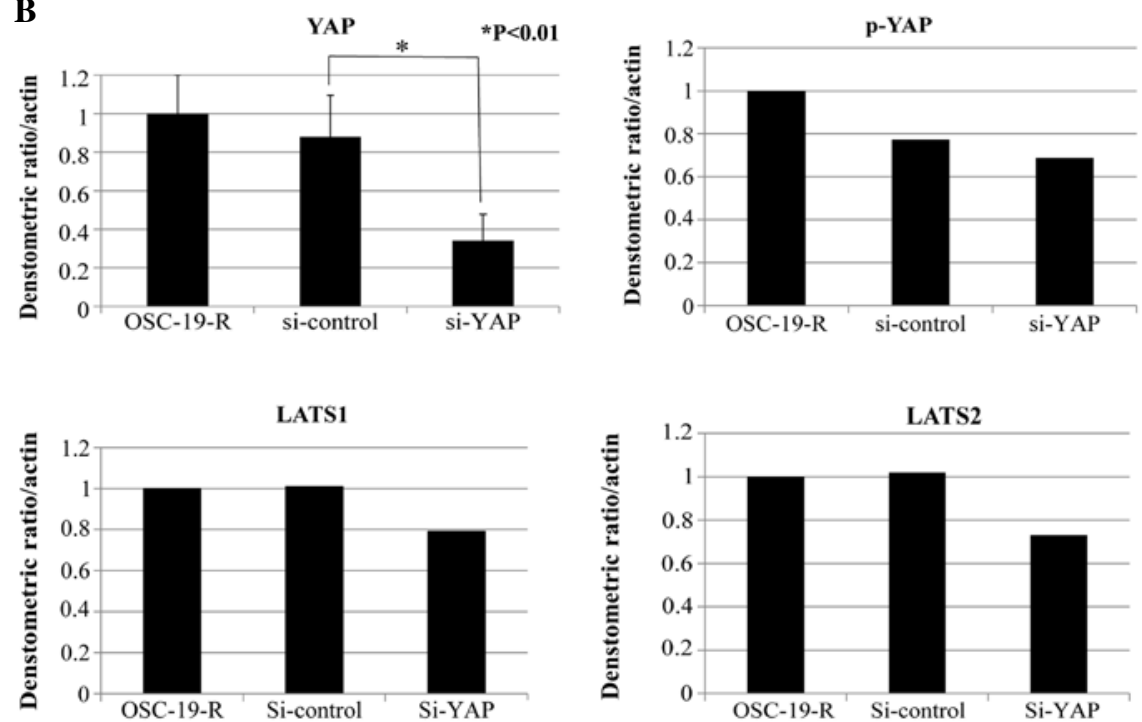

$\mathbf{C}$

siControl

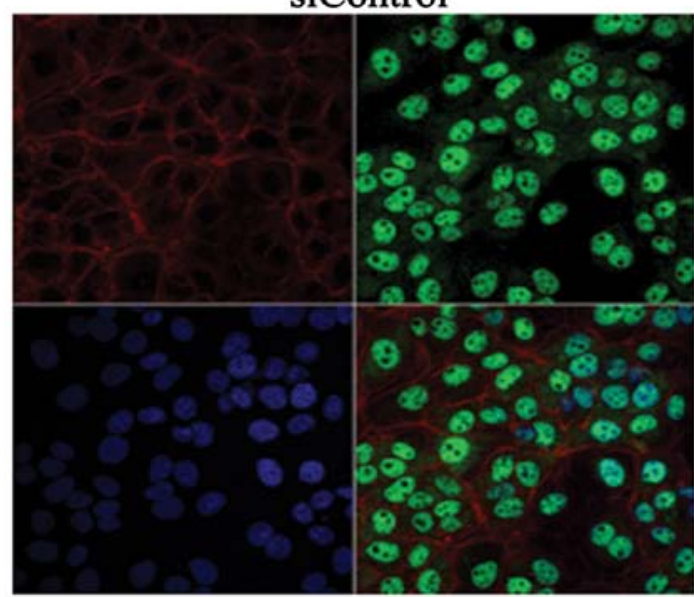

siYAP

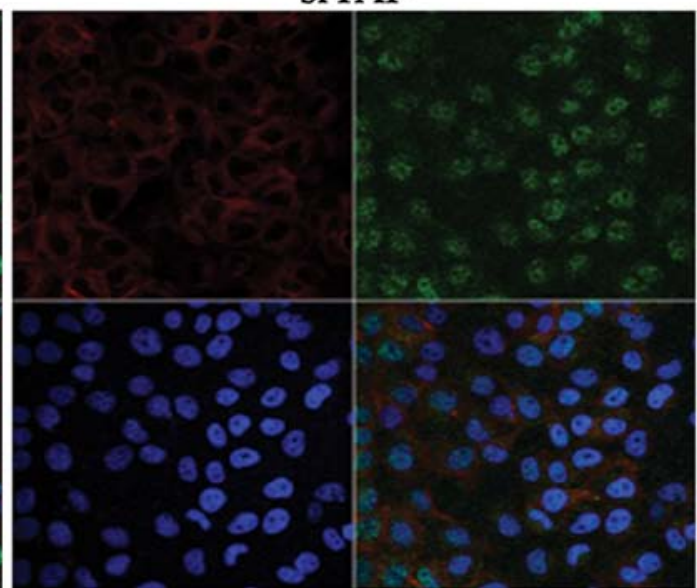

Figure 4. (A and B) Following treatment with YAP-specific siRNAs, we observed a dramatic reduction of YAP expression. The expression level of phosphoYAP and LATS1/2 were uninhibited. (C) Immunocytochemical analysis showed that YAP-specific siRNA-treated OSC-19-R cells revealed a reduction in the extent of YAP nuclear translocation. 
There was a significant association for each $\mathrm{IC}_{50}$ value between parental and CDDP-resistant cell lines.

Western blotting of ATP7B protein levels. The CDDP-resistant cell lines, SCCKN-R and HSC-3-R, showed overexpression of the ATP-7B protein (Fig. 1B). There were no obvious difference in expression levels of GST- $\pi$ and ERCC1 between parental and CDDP-resistant cell lines. We did not observe any difference in expression levels of the apoptotic cascade protein caspase-3/7 in OSC-19 and OSC-19-R cells (data not shown).

A new mechanism involving YAP induced CDDP resistance. Compared with parental OSC-19 cells, OSC-19-R cells were obviously larger (Fig. 2A). We investigated the Hippo pathway as it is known to regulate organ size. Expression levels of YAP were not significantly different in the OSC-19 and OSC-19-R cell lines, however, expression levels of phosphorylated YAP in OSC-19-R cells were decreased (Fig. 2B and C). In addition, there was no difference in CD44, TEAD, P73, AKT, or phosphorylated AKT expression levels. While high expression levels of LATS1/2 were revealed, our results indicated that expression levels of non-phosphorylated YAP, a substrate of LATS1/2, were decreased. Our immunocytochemistry results revealed that YAP translocated from the cytoplasm to the nucleus in OSC-19-R cells (Fig. 3).

YAP nuclear translocation inhibited by siRNAs. To clarify the translocation of YAP in CDDP-resistant cells, knockdown experiments using siRNAs were conducted. We observed a dramatic reduction of YAP expression in YAP-specific siRNA-treated cells compared with cells treated with control siRNAs using western blotting. Expression levels of phosphoYAP and LATS1/2 were not inhibited by siRNA treatments (Fig. 4A and B). Our immunocytochemistry results confirmed that expression levels of YAP were reduced in YAP-specific siRNA-treated cells (Fig. 4C). The OSC-19-R cells treated with YAP-specific siRNAs showed a reduction in YAP nuclear translocation.

Overcoming acquired CDDP resistance with YAP siRNAs. Nuclear translocation of YAP appeared to induce acquired CDDP resistance in the OSC-19 cell line. Treatment with YAP-specific siRNAs ameliorated acquired CDDP resistance, with sensitivity to CDDP increased in OSC-19-R cells transfected with siYAP, and an $\mathrm{IC}_{50}$ reduction rate of $38.0 \%$ calculated (Fig. 5A). Statistically, there was no difference between the OSC-19 and OSC-19-R cells as shown by growth curves, but there was obviously difference between OSC-19R-siControl and OSC-19-R-siYAP cells treated with CDDP (Fig. 5B). Proliferation of OSC-19-R-siYAP cells was significantly inhibited by CDDP from day 2 onwards.

\section{Discussion}

Many studies have been conducted to investigate mechanisms of acquired resistance in tumor cells to CDDP (18). The molecular mechanisms that underlie CDDP resistance are not well understood. From our results in the present study, we propose a new mechanism of acquired resistance to CDDP
A

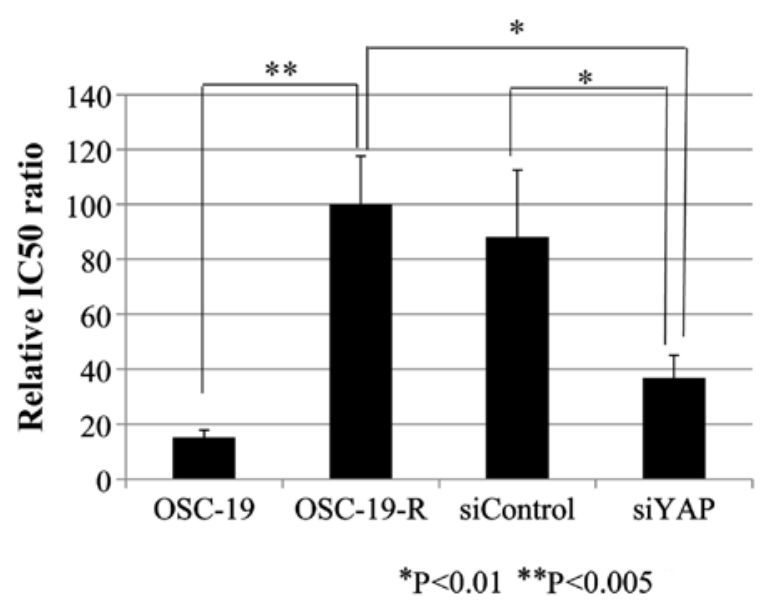

B
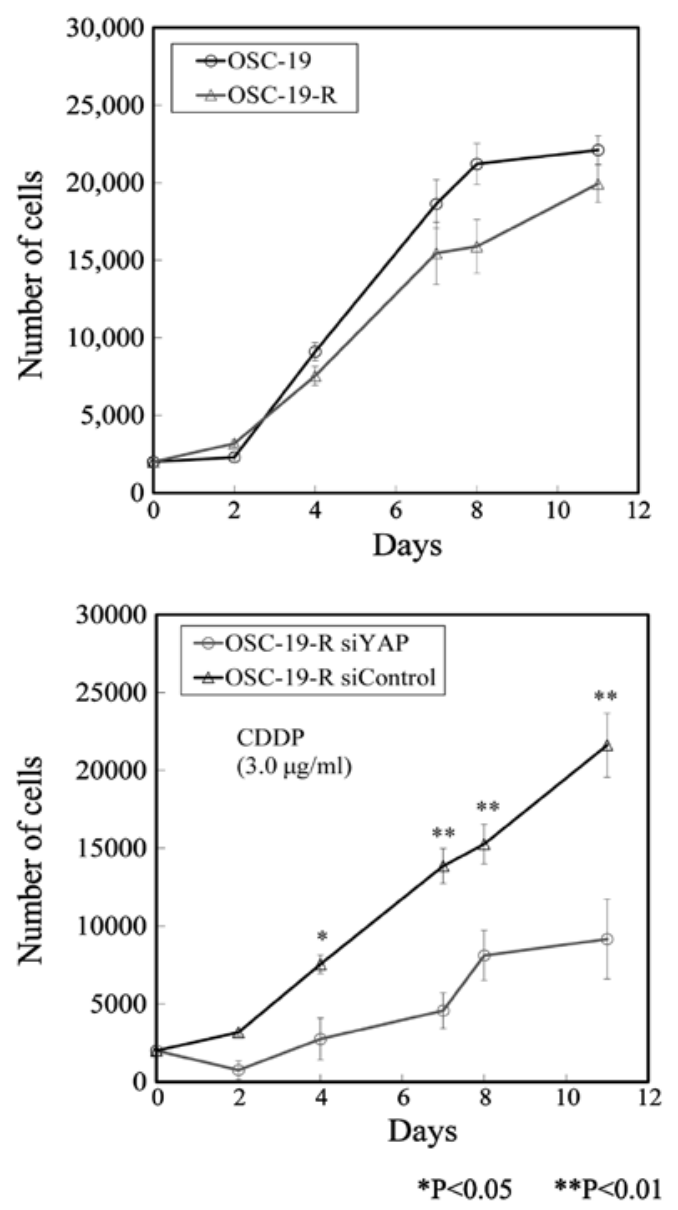

Figure 5. (A) Treatment of cells with YAP-specific siRNAs allowed cells to recover from acquired CDDP resistance, with sensitivity to CDDP significantly increased in OSC-19-R-siYAP cells. (B) Compared with OSC-19-R-siControl cells, proliferation of OSC-19-R-siYAP cells was significantly inhibited by CDDP treatment from day 2 onwards.

that is related to the Hippo pathway. The Hippo pathway controls organ size in diverse species, and dysregulation of this pathway can induce tumors (19). To the best of our knowledge, we are the first to report that translocation of YAP induced acquired resistance to CDDP in OSCC, and that repression of YAP expression with siRNAs increased sensitivity to CDDP in cells that have acquired CDDP resistance.

We established three CDDP-resistant cell lines, with two of these cell lines exhibiting higher expression levels of 
ATP7B than those of other molecules when they acquired CDDP resistance. With respect to CDDP transport, CTR1 and ATP7B influence its uptake and efflux, respectively. ATP7B is a $\mathrm{Cu}^{2+}$-ATPase, with these enzymes known to be homologous in structure and function, and able to mediate the efflux of $\mathrm{Cu}^{2+}(20)$. The major $\mathrm{Cu}^{2+}$ uptake transporter is copper transporter 1 (CTR1). Recent results have shown that the $\mathrm{Cu}^{2+}$ homeostasis system also regulates the uptake, intracellular compartmentalization and efflux of CDDP (20-22). It was reported that cell lines acquired resistance to CDDP due to overexpression of ATP7B, resulting in greater efflux of CDDP (23). Based on the finding that overexpression of ATP7B induced acquired resistance to CDDP in OSCC, we ensured that ATP7B was overexpressed in the SCCKN-R and HSC-3-R cell lines we established. The previous study (23) also reported overexpression of ATP7B in CDDP-resistant OSC-19 cells they generated, however, we did not observe this in our OSC-19-R cells. Moreover, the $\mathrm{IC}_{50}$ of OSC-19-R was $30.6 \mu \mathrm{M}(23)$, which is about one-third the level of that determined in our present study. We postulate that this discrepancy is a result of the difference in how the resistance was acquired. Dose escalation of CDDP was more harsh in the previous report compared with the method we used (23). Under clinical situations, high dose CDDP chemotherapy, which is an example of intra-arterial super-selective chemotherapy, is widly and safely used on a daily basis. Therefore, we sought to establish CDDP-resistant cell lines that were dependent upon higher fixed concentrations of CDDP exposure.

Although much progress has been made towards our understanding of the role of the Hippo pathway with regards to tumorigenesis, organ size control, and stem cell renewal and differentiation (24-27), its function(s) with respect to chemotherapeutic drug response is largely unknown (28). In human HNSCC and OSCC lines, amplification of the chromosomal region that encodes YAP, 11q21-22, is frequently seen $(29,30)$. Reports also exist showing that core components of the Hippo pathway might be involved in the response of cancer cells to chemotherapeutic drugs such as paclitaxel $(16,31-34)$. In the present study, we established YAP as an important protein for CDDP drug resistance, indicating a strong link between EMT and drug resistance (35). It is possible that phosphorylation and inactivation of YAP might compromise its ability to trans-activate another transcription factor that suppresses E-cadherin and induces EMT. Moreover, because EMT plays important roles in stem cell renewal, cell migration, anoikis, invasion, and metastasis $(16,36,37)$, it would also be interesting to further explore how phosphorylation of YAP under physiological condition regulates these processes.

In conclusion, our results revealed a new mechanism for YAP, which could be used as a molecular target to overcome acquired resistance of CDDP. Further examination of the relationship between YAP levels, translocation, and phosphorylation status of YAP, and the survival of clinical cancer patients before and after treatment with CDDP, are required to sufficiently determine if YAP and phospho-YAP can be used as prognostic biomarkers for predicting sensitivity to CDDP. It is possible YAP and phospho-YAP could be potential therapeutic targets for the treatment of cancer patients that are drug-resistant.

\section{Acknowledgements}

The authors thank Dr Keiko Wakai for preparation of the experiments and Ms. Takako Nanba for management of grants. This study was supported by Grants-in-Aid for JSPS KAKENHI (grant nos. 25463123 to K.N. and 26861760 to K.Y.).

\section{References}

1. Haddad RI and Shin DM: Recent advances in head and neck cancer. N Engl J Med 359: 1143-1154, 2008.

2. Overgaard J, Mohanti BK, Begum N, Ali R, Agarwal JP, Kuddu M, Bhasker S, Tatsuzaki H and Grau C: Five versus six fractions of radiotherapy per week for squamous-cell carcinoma of the head and neck (IAEA-ACC study): A randomised, multicentre trial. Lancet Oncol 11: 553-560, 2010.

3. Roepman P, Wessels LF, Kettelarij N, Kemmeren P, Miles AJ, Lijnzaad P, Tilanus MG, Koole R, Hordijk GJ, van der Vliet PC, et al: An expression profile for diagnosis of lymph node metastases from primary head and neck squamous cell carcinomas. Nat Genet 37: 182-186, 2005.

4. Weaver A, Fleming S, Ensley J, Kish JA, Jacobs J, Kinzie J, Crissman J and Al-Sarraf M: Superior clinical response and survival rates with initial bolus of cisplatin and 120 hour infusion of 5-fluorouracil before definitive therapy for locally advanced head and neck cancer. Am J Surg 148: 525-529, 1984.

5. Rooney M, Kish J, Jacobs J, Kinzie J, Weaver A, Crissman J and Al-Sarraf M: Improved complete response rate and survival in advanced head and neck cancer after three-course induction therapy with 120-hour 5-FU infusion and cisplatin. Cancer 55: 1123-1128, 1985

6. Wang D and Lippard SJ: Cellular processing of platinum anticancer drugs. Nat Rev Drug Discov 4: 307-320, 2005.

7. Hall MD, Okabe M, Shen DW, Liang XJ and Gottesman MM: The role of cellular accumulation in determining sensitivity to platinum-based chemotherapy. Annu Rev Pharmacol Toxicol 48: 495-535, 2008.

8. Shen DW, Pouliot LM, Hall MD and Gottesman MM: Cisplatin resistance: A cellular self-defense mechanism resulting from multiple epigenetic and genetic changes. Pharmacol Rev 64: 706-721, 2012.

9. Avruch J, Zhou D, Fitamant J and Bardeesy N: Mst1/2 signalling to Yap: Gatekeeper for liver size and tumour development. Br J Cancer 104: 24-32, 2011.

10. Wang K, Degerny C, Xu M and Yang XJ: YAP, TAZ, and Yorkie: A conserved family of signal-responsive transcriptional coregulators in animal development and human disease. Biochem Cell Biol 87: 77-91, 2009.

11. Yuan M, Tomlinson V, Lara R, Holliday D, Chelala C, Harada T, Gangeswaran R, Manson-Bishop C, Smith P, Danovi SA, et al: Yes-associated protein (YAP) functions as a tumor suppressor in breast. Cell Death Differ 15: 1752-1759, 2008.

12. Vidal M and Cagan RL: Drosophila models for cancer research. Curr Opin Genet Dev 16: 10-16, 2006.

13. Nylander K, Coates PJ and Hall PA: Characterization of the expression pattern of p63 alpha and delta Np63 alpha in benign and malignant oral epithelial lesions. Int J Cancer 87: 368-372, 2000.

14. Lo Muzio L, Santarelli A, Caltabiano R, Rubini C, Pieramici T, Trevisiol L, Carinci F, Leonardi R, De Lillo A, Lanzafame S, et al: p63 overexpression associates with poor prognosis in head and neck squamous cell carcinoma. Hum Pathol 36: 187-194, 2005.

15. Ge L, Smail M, Meng W, Shyr Y, Ye F, Fan KH, Li X, Zhou HM and Bhowmick NA: Yes-associated protein expression in head and neck squamous cell carcinoma nodal metastasis. PLoS One 6: e27529, 2011.

16. Zhao Y, Khanal P, Savage P, She YM, Cyr TD and Yang X: YAP-induced resistance of cancer cells to antitubulin drugs is modulated by a Hippo-independent pathway. Cancer Res 74: 4493-4503, 2014.

17. Yamamura M, Noguchi K, Nakano Y, Segawa E, Zushi Y, Takaoka K, Kishimoto H, Hashimoto-Tamaoki T and Urade M: Functional analysis of Zyxin in cell migration and invasive potential of oral squamous cell carcinoma cells. Int J Oncol 42: 873-880, 2013. 
18. Köberle B, Tomicic MT, Usanova S and Kaina B: Cisplatin resistance: Preclinical findings and clinical implications. Biochim Biophys Acta 1806: 172-182, 2010.

19. Liu AM, Wong KF, Jiang X, Qiao Y and Luk JM: Regulators of mammalian Hippo pathway in cancer. Biochim Biophys Acta 1826: 357-364, 2012.

20. Solioz M and Vulpe C: CPx-type ATPases: A class of P-type ATPases that pump heavy metals. Trends Biochem Sci 21: 237-241, 1996.

21. Culotta VC, Lin SJ, Schmidt P, Klomp LW, Casareno RL and Gitlin J: Intracellular pathways of copper trafficking in yeast and humans. Adv Exp Med Biol 448: 247-254, 1999.

22. Katano K, Safaei R, Samimi G, Holzer A, Rochdi M and Howell SB: The copper export pump ATP7B modulates the cellular pharmacology of carboplatin in ovarian carcinoma cells. Mol Pharmacol 64: 466-473, 2003.

23. Samimi G, Katano K, Holzer AK, Safaei R and Howell SB Modulation of the cellular pharmacology of cisplatin and its analogs by the copper exporters ATP7A and ATP7B. Mol Pharmacol 66: 25-32, 2004.

24. Safaei R and Howell SB: Copper transporters regulate the cellular pharmacology and sensitivity to Pt drugs. Crit Rev Oncol Hematol 53: 13-23, 2005.

25. Yoshizawa K, Nozaki S, Kitahara H, Ohara T, Kato K, Kawashiri S and Yamamoto E: Copper efflux transporter (ATP7B) contributes to the acquisition of cisplatin-resistance in human oral squamous cell lines. Oncol Rep 18: 987-991, 2007.

26. Hong W and Guan KL: The YAP and TAZ transcription co-activators: Key downstream effectors of the mammalian Hippo pathway. Semin Cell Dev Biol 23: 785-793, 2012.

27. Sudol M and Harvey KF: Modularity in the Hippo signaling pathway. Trends Biochem Sci 35: 627-633, 2010.
28. Yu FX and Guan KL: The Hippo pathway: Regulators and regulations. Genes Dev 27: 355-371, 2013.

29. Guo $X$ and Zhao B: Integration of mechanical and chemical signals by YAP and TAZ transcription coactivators. Cell Biosci 3: 33,2013

30. Lai D, Visser-Grieve $S$ and Yang X: Tumour suppressor genes in chemotherapeutic drug response. Biosci Rep 32: 361-374, 2012.

31. Carey TE, Van Dyke DL and Worsham MJ: Nonrandom chromosome aberrations and clonal populations in head and neck cancer. Anticancer Res 13 (6B): 2561-2567, 1993.

32. Snijders AM, Schmidt BL, Fridlyand J, Dekker N, Pinkel D, Jordan RC and Albertson DG: Rare amplicons implicate frequent deregulation of cell fate specification pathways in oral squamous cell carcinoma. Oncogene 24: 4232-4242, 2005.

33. Lai D, Ho KC, Hao Y and Yang X: Taxol resistance in breast cancer cells is mediated by the hippo pathway component TAZ and its downstream transcriptional targets Cyr61 and CTGF. Cancer Res 71: 2728-2738, 2011.

34. Visser S and Yang X: Identification of LATS transcriptional targets in HeLa cells using whole human genome oligonucleotide microarray. Gene 449: 22-29, 2010.

35. Kawahara M, Hori T, Chonabayashi K, Oka T, Sudol M and Uchiyama T: Kpm/Lats2 is linked to chemosensitivity of leukemic cells through the stabilization of p73. Blood 112: 3856-3866, 2008.

36. Cordenonsi M, Zanconato F, Azzolin L, Forcato M, Rosato A, Frasson C, Inui M, Montagner M, Parenti AR, Poletti A, et al: The Hippo transducer TAZ confers cancer stem cell-related traits on breast cancer cells. Cell 147: 759-772, 2011.

37. Frisch SM, Schaller M and Cieply B: Mechanisms that link the oncogenic epithelial-mesenchymal transition to suppression of anoikis. J Cell Sci 126: 21-29, 2013. 